\title{
WARUM VERWENDEN MARKETINGSTRATEGEN GERNE AUS DEM MILITÄRISCHEN BEREICH ENTLEHNTE WÖRTER UND REDEWENDUNGEN?
}

\author{
VERSUCH ÜBER MIMESIS UND DEN SLOGAN WHY DO \\ MARKETING PROFESSIONALS USE MILITARY SLANG?
}

\author{
Monika Mühlpfordt ${ }^{1}$ \\ Recebido em: 02/2018 \\ Aprovado em: 03/2018
}

\begin{abstract}
Zusammenfassung: Traditionell wird Sprache im Krieg in der Gestalt des Schlachtrufes eingesetzt. Im schottischen Gälisch heißt er sluagh gairm, und daraus wurde der Slogan. Der Name einer Formel, die beim Gebrauch auf dem Schlachtfeld sowohl die eigenen Leute motivieren als auch den Gegner einschüchtern soll, ist in den vergangenen Jahrzehnten zum Sammelbegriff für mehr oder weniger erfolgreiche Versuche einer Verführung zum Geldausgeben geworden. Auch von Kampagnen, also Feldzügen, sprechen Werbefachleute ganz selbstverständlich. Wieso ist dieser verbal-blutige Ernst nötig in einem Bereich, in dem es gar nicht um Leben und Tod geht? Was Menschen anscheinend schon immer wertvoll genug war, um ihr eigenes Blut dafür zu vergießen, ist die menschengemachte Ordnung - ein Regelwerk, mit der eine Gruppe von Menschen die Unberechenbarkeit ihrer Welt in Schach hält. Derartige Ordnungen wurden immer schon einer höheren Instanz gewidmet, die ihre Anhänger vom bösen Unwägbaren erlösen sollte. Mit Letzterem sind heute die Aktienkurse und Konsumentenlaunen gemeint, gegen deren Willkür eine starke, einzigartige Markenidentität die unter ihr Subsumierten in Schutz nehmen soll, welche daraufhin von diesen wiederum mit rückhaltloser Hingabe verteidigt wird.
\end{abstract}

\begin{abstract}
Battle cries are a traditional manifestation of language in times of war. In Scottish-Gaelic, they were called "sluagh gairm", from which the word slogan emerged. In a war cry, special wording motivates fellow combatants, while at the same time scaring the enemy. Today's advertising slogans, comparatively minor, are expected to tempt consumers into spending some money. Quite naturally, advertising experts use the term "campaign", which literally labels a military expedition. Why do we make it sound deadly serious when it is not really a matter of life and death? However, defense of man-made regulations always seemed to be worth bloodshed in history: ritual and public orders used by humans to keep the world's unpredictability at bay were of utmost significance. Those regulations in former times, devoted to a higher authority, a spirit who was expected to grant deliverance from the evils of uncertainty. Today, often stock prices or consumer attitudes are meant by these threats. Powerful brand identities could be seen as protective deities against harmful influences and thus are defended fiercely.
\end{abstract}

\footnotetext{
${ }^{1}$ Dr. Monika Mühlpfordt, geboren 1978, studierte ab 2002 Kommunikationsdesign in Mannheim und Zürich, Masterabschluss 2009. Promotion 2016 im Bereich Medientheorie und philosophische Ästhetik an der Hochschule für Grafik und Buchkunst Leipzig. E-mail: monika muehlpfordt@web.de
} 
„Am Schluss, über meiner Unterschrift und anstelle eines traditionellen ,Danke für Ihre Aufmerksamkeit‘ oder ,Mit freundlichen Grüßen` schrieb ich ,Onward‘. Bis heute bin ich nicht sicher, ob ich das Wort schon gebraucht habe, bevor ich das Memo schrieb. Aber in jenem Augenblick fiel es mir ein, und es passte genau. Ich spürte, dass es richtig war, es war ein Schlachtruf, der zu dem gewaltigen, aufregenden Abenteuer zu passen schien, zu dem sich unser kleines Unternehmen aufmachte.“

(Schultz 2011, S. 30 f.)

So schreibt Howard Schultz in seiner Funktion als Vorstandsvorsitzender von Starbucks im Buch Onward. How Starbucks Fought for Its Life without Losing Its Soul über sein erstes Memo an die damaligen Mitarbeiter einer einzigen Kaffee-Bar namens Il Giornale, die das italienische Kaffeepausengefühl in die Vereinigten Staaten holen sollte.

Onward — zu Deutsch: „Vorwärts“, hier vor allem in Verbindung mit „-marsch!“ militärisch konnotiert, aber auch ein Name demokratisch-oppositioneller Wochenzeitungen, kaiserlicher Torpedoboote und Armeesportvereinigungen des 19. und teilweise noch 20. Jahrhunderts — von dieser knappen Aufforderung ist also bekannt, dass sie für Schultz die Bedeutung eines Schlachtrufes hat. Und nicht nur im Titel seines Buches liest man vom Kampf auf Leben und Tod. Schultz verwendet über die gesamte Publikation hinweg militärische Einsprengsel wie „Wunderwaffe“, „schwierige Schlacht“, „als ob unser Leben vom Sieg abhinge“, „an vorderster Front“, ,im Schulterschluss kämpfen“ und fürchtet Konkurrenten, die „unsere Gäste erobern“ könnten; das alles im Zusammenhang mit Unternehmensführung und Marketing. Und er ist damit nicht allein: ein Marketingratgeber von Hermann H. Wala gibt Tipps zum „Erobern eines Logenplatzes im Kundenkopf“ und weiß, „Feindbilder haben schon immer geholfen, die eigenen Reihen fester zu schließen“. Die Marketingstrategin Karen Heumann sagt in einem Interview, sie „fände es sogar klüger, wenn Agenturen nicht ständig gegeneinander in die Schlacht geschickt würden“"

Ist es ein neues Phänomen, dass das Führen eines Unternehmens, das Vermarkten von Produkten oder der dafür nötigen kreativen Arbeitskraft die Beteiligten an einen Kriegszustand erinnert? Möglich wäre das, angesichts der Häufigkeit, mit der von steigender Konkurrenz, krisengebeutelten Märkten, undurchdringlicher bereits vorhandener Reizüberflutung aufseiten des umworbenen Kunden die Rede ist. Aber der „Krieg“ haftet der Kommunikation zwischen Markenherstellern und Verbrauchern schon immer an: warum sonst nennt man werbestrategische Maßnahmen Kampagne, wie einen Feldzug?

Das Wort Slogan, das nicht nur im angelsächsischen Sprachraum seit vielen Jahrzehnten 
die zur Werbung gehörigen Aufforderungen, anpreisenden Kommentare und Qualitätsversprechen bezeichnet (wenn auch zur Zeit unter Werbern etwas aus der Mode geraten) kommt von den Wörtern für „Volk“ und „Ruf“, sluagh gairm, im schottischen Gälisch, wo es einen Ruf meinte, der die Angehörigen eines Clans auf dem Schlachtfeld versammeln sollte. Einen Schlachtruf also.

Zunächst einmal ist Krieg ja eine bewaffnete Auseinandersetzung zwischen mindestens zwei Kollektiven, bei der eine Gruppe von Mitgliedern stellvertretend für das gesamte Kollektiv zur Verteidigung, zur Erringung wirtschaftlicher Vorteile oder zur Vergrößerung des eigenen Gebietes ihr Leben aufs Spiel setzt. Heute scheint sich die Erklärung der Beweggründe für solche verlustreichen Auseinandersetzungen weit schwieriger $\mathrm{zu}$ gestalten als noch vor wenigen Jahrhunderten. Aber um die heutigen soll es hier auch weniger gehen - das Vokabular, das von „Nahkampf“ und „geschlossenen Reihen“ spricht, bezieht sich nämlich recht deutlich auf die Vorstellung eines traditionellen Schlachtfeldes, ohne Angriffe aus der Luft oder gar den Einsatz intelligenter Gefechtssysteme und unbemannter Kampfroboter. Die vom Marketing beibehaltenen oder aufgewärmten Ausdrücke lassen vor dem inneren Auge einen Feldherrn seine tapferen Mannen zu Fuß oder zu Pferd losschicken, wobei er ihnen etwas zuruft, das sie ermutigen und für das Vorhaben des Feldherrn begeistern soll. Weil von diesen „alten“ Schlachten nur Geschichtsbücher und Reiterstandbilder übriggeblieben sind, denkt man jedoch gemeinhin in diesem Kontext nicht etwa an von Bajonetten durchbohrte Tote und Sterbende und stellt sich auch nicht vor, es seien eigene Familienmitglieder darunter. Sonst könnte das Kriegsvokabular wohl kaum in zivilen Zusammenhängen gebraucht werden, ohne dass es schmerzt. Man soll lediglich „ganz, ganz ernst“ assoziieren. Ein kriegerischer Ernstfall muss immerhin als ernst genug empfunden werden, dass man sich dafür gegenseitig in großer Zahl abschlachtet. „Gegenseitig“ sagt bereits, dass ein solches Vorgehen erst einmal eine deutlich als solche erkennbare Gegenseite, eine gegnerische Gruppe, voraussetzt.

In der italienischen Nationalhymne heißt es noch heute „Lasst uns die Reihen schließen, // Wir sind bereit zum Tod“, in der türkischen kommt „unser vergossenes Blut“ vor, in der brasilianischen „,... sterben wir in Ehren“. Dass Nationalhymnen heute noch zum Beispiel vor Fußball-Länderspielen gesungen werden, ist ein Rest aus den Kriegstagen, in denen die Kämpfe „Mann gegen Mann“, was der Fußball spielerisch nachahmt, ausgetragen wurden, wozu man vorher sang und Slogans skandierte (wie übrigens heutige Cheerleaders das auch noch tun), um sich Mut zu machen. Schlachtrufe sollen dabei helfen. Ein solcher wie „Vorwärts“ ist lediglich eine Ergänzung zu einer vorausgegangenen ausführlichen Rede, eine Art Startschuss. Die 
Schlachtrufe der heutigen Bundeswehr sind ebenfalls knapp und dienen wohl nur dazu, ein fertig eingedrilltes Programm ablaufen zu lassen und Hemmungen in Grund und Boden zu schreien. So wie der Ruf „Panzergrenadiere!“, auf den man mit „Dran! Drauf! Drüber!“ antwortet, und „Ohne Furcht!“ — „Nach vorn!“ der Heeresflieger, dessen zweiter Teil an „Vorwärts“ oder „Onward“ erinnert.

Die aussagekräftigeren Schlachtrufe, als deren Langversionen sich viele Nationalhymnen enttarnen lassen, beschwören die Liebe zur eigenen Nation und die Bereitschaft, diese durch den eigenen Tod unter Beweis zu stellen. Man opfert sich sozusagen für eine Grenze, innerhalb derer man „die eigenen Leute“ verortet und die man in Form einer Front sinnbildlich verteidigt. Innerhalb der um „uns“ gezogenen Grenze herrschen unsere Sprache und unsere Regeln, Rechte, Pflichten und Gesetze. Diese können Menschen also derart heilig sein, dass dafür getötet und gestorben wird.

Vieles spricht dafür, dass nicht zuerst die Ordnung da war, die das Töten rechtfertigt, sondern dass das Töten, in Form von Opferritualen, umgekehrt die erste Form einer Ordnung losgetreten haben muss: einschneidende Erlebnisse im Leben von Menschen sind Geburt und Tod, dicht gefolgt von Erlebnissen, die nah an diesen beiden „Rändern“ liegen — zum Beispiel wäre das das Miterleben des Todes eines anderen, das Gebären der eigenen Kinder, das Gefühl, knapp dem Tod entronnen zu sein und so weiter. Christoph Türcke (2009, S. 62) dazu:

„Das griechische Verb rezein [...] bedeutet sowohl ,Opfer darbringen“ als auch generell ,handeln, tätig sein' und drückt damit aus, daß Opfern der Inbegriff menschlichen Handelns, die menschenspezifische Tätigkeit schlechthin ist."

Das Darbringen eines Opfers bleibt im Gedächtnis. Es konnte wiederkehrende Sequenzen, Strukturierungseinheiten, also Abschnitte, etablieren, die zur Linderung des Schmerzes durch Einschnitte wie Hungersnöte, Naturkatastrophen und Angriff wilder Tiere beitrugen, allerdings nur, indem Menschen selbst anderen, mehr oder weniger verwandten, Lebewesen tödliche Schnitte beibrachten.

Der Ordnung von Leben und Tod im Naturkreislauf setzen Menschen seit jeher eine Ordnung auf eigene Faust entgegen. Diese ist zum einen zunächst mimetisch, nämlich solange lediglich getötet wird (ohne das dieser Vorgang schon als "Opfern" bezeichnet werden könnte), also verwandt mit der Fähigkeit des Tieres, noch ganz ohne Empathie mit Zähnefletschen auf das Zähnefletschen des Gegenübers zu reagieren; andererseits bringt es eine neue Qualität in die Welt: erst jetzt können menschliche Unternehmungen menschliche Schicksale beeinflussen. 
Dem Slogan im ersten Wortsinn, dem sluagh gairm, ist die Verteidigung einer Ordnung, zu der er aufruft, allerdings nicht unmittelbar anzusehen. Von 40 Slogans, die bei den Clans der schottischen Highlands in Gebrauch waren (und es heute zum Beispiel bei Highland Gatherings noch immer sind), beziehen sich 17 auf Landschaftsmerkmale wie einen Wald oder einen Felsen. Zum Beispiel ist der Ruf des Clans MacGregor „Ard-coille“, was „Hochwald“ bedeutet, die Macphersons hören auf „Creag an Dhubh“, „Schwarzer Felsen“. Zählt man zu den Landschaftsslogans auch noch Slogan gewordene Namen von Burgen hinzu, kommt man schon auf 23, womit über die Hälfte aller Highland-Slogans sich lediglich auf bestimmte Orte beziehen, ohne weitere Aussage. Die Vermutung wäre übrigens nicht abwegig, dass die Namen menschengemachter Bauwerke in Slogans jünger sind als die „natürlichen“, denn der FraserClan verwendete „A ’Mhór-fhaiche“, „Das große/bedeutende Feld“, als Slogan, der dann später durch den Namen einer Burg ersetzt wurde. Die restlichen der 40 Slogans aus dem HighlandBereich liegen recht weit auseinander, was ihre Bedeutungen angeht: es gibt solche, die man unmittelbar als Schlachtrufe erkennt wie „Bàs no Beatha“ — „Tod oder Leben“, „Buaidh no Bàs, was „Sieg oder Tod“ bedeutet, oder „Cuimhnich bàs Ailpein“, „Denkt an den Tod Alpins!“‘, das wie ein Aufruf dazu klingt, den Tod des ersten Königs von Schottland, Kenneth I. MacAlpin, zu rächen. Bei den MacArthurs wird um Aufmerksamkeit gebeten: „Eisd! O Eisd!“ heißt „Hört! O hört!““, und ein weiterer Clan spricht die Warnung aus, man sei „Garg ’n uair dhùisgear“, in etwa: „Grimmig, wenn gereizt“. Aber diese thematisch verstreuten, für heutige Ohren aussagekräftigeren Slogans kommen zahlenmäßig gegen die „Orte“ nicht an.

Was bedeutet es, wenn der Name eines Landschaftmerkmals für die eigene Gesellschaftsordnung steht, von der eine Gruppe zusammengehalten wird? Der Name eines Berges als Schlachtruf hat natürlich Gemeinsamkeiten mit dem Dom als Wahrzeichen für Köln, dem Eiffelturm, zu dem man „Paris“ assoziiert, und so weiter: die zu verteidigende Weltordnung ist mit einem Platz in der Welt verknüpft, den man von Weitem an signifikanten Merkmalen erkennt. Aber während man sich in eine Schlacht stürzt, die zu überleben oft unwahrscheinlich genug ist, reicht als Motivationsschrei sicher nicht „Ihr wisst schon, wir sind die, die am Fuß des Berges wohnen“ (zumal bei einem Kampf zu Verteidigungszwecken der Berg wahrscheinlich in Sichtweite ist). Die Bezugnahme auf eine bestimmte Landschaft im Slogan muss eine weit gewichtigere Bedeutung haben: Sie könnte an kultisch aufgeladene Orte erinnern, deren Kraft man sich zunutze macht, während man auf den Feind zustürmt oder sich zum Angriff versammelt. Die Ordnung, die von einem Kollektiv verteidigt wird, hat ja ihre Wurzeln in einem blutigen Untergrund, und dieser ist nicht irgendwo - es gehört ein fester, 
definierter Kultplatz dazu. Eine Ritualordnung schreibt nicht ohne Grund vor, an welchem Ort das Opfer darzubringen ist - spätestens, wenn aus Kultordnungen auch Alltags-Ordnungen hervorgehen, müssen beide nicht nur zeitlich (das äußert sich noch heute in religiösen Feiertagen und bei manchen Ethnien in einem Nichtangriffspakt während mehrtägiger Kulthandlungen), sondern auch räumlich voneinander getrennt werden.

Ritualorte sind mit der Kraft eines ungeheuerlichen Geschehens aufgeladen, noch in weit größerem Maße, als es in Gespenstergeschichten ein Spukhaus wäre - eher vergleichbar vielleicht mit der Kraft der alten Indianerfriedhöfe in Romanen von Stephen King, mit denen er großen kommerziellen Erfolg hatte, weil der Schrecken für Menschen auch eine Anziehungskraft hat. Diese ist verwandt mit dem traumatischen Wiederholungszwang, dem das Opfern gehorchte (vgl. Türcke 2009, S.59 ff.), wenn es den Schrecken mit ebenso schrecklichen Mitteln bekämpfte. Es muss sich für die Beteiligten so angefühlt haben, als könnte man den „Teufel“ der bedrohlichen Naturkräfte ringsherum nur durch den „Beelzebub“ eigener Gewaltanwendung austreiben.

Das Gefühl einer „höheren Macht“, die sich durch das Zusammenwirken Vieler ergab, und der Schmerz, den die eigene Tat verursachte, führten zum Imaginieren eines Adressaten, der das Opfer verlangt, als eines ersten Gegenübers. Diese erste Vorstellung einer gemeinsamen Geistererscheinung könnte man auch, aus begründetem Anachronismus, „Teamgeist“ nennen - einen Spuk, der da erscheint, wo man sich in einem bestimmten mentalen Zustand versammelt, wie es auch bis heute die Art vieler Gottheiten ist (Matthäus 18, 20: „Denn wo zwei oder drei versammelt sind in meinem Namen, da bin ich mitten unter ihnen“). Nur die Macht, die beim Opfern erscheint, kann Seelenfrieden einkehren lassen. Deshalb gilt das, was das Schrecklichste verlangt, gleichzeitig als die einzige Stelle, bei der man Schutz suchen kann - die ihrerseits durch das Einhalten von Regeln um jeden Preis geschützt werden muss.

Später übernimmt oft die Nation die Rolle einer solchen Schutzmacht: wenn ihr Schaden zugefügt wird, geht es uns allen an den Kragen. Die Verteidigung der Nation ist dann auch von Gesängen und Beschwörungen begleitet, die sich wie in der verniedlichenden Bezeichnung „Ohrwurm“ ins Gedächtnis eingraben. Ohne derartige Einfleischungen würde auch kein Ritual auskommen. Diese akustische Verwandtschaft könnte ein Grund dafür sein, warum viele gälische Slogans im Krieg einen Ort beschwören: wir befinden uns zwar auf dem Schlachtfeld, aber dort ist Es über uns gekommen (ähnlich reden heutige Wagner-Dirigenten über den Orchestergraben im Bayreuther Festspielhaus). Vielleicht können die übermenschlichen, weil gemeinsamen, Kräfte aus einem Ritualvollzug mobilisiert und an einen anderen Ort geholt 
werden, wenn man den Ort ihres üblichen Auftretens ausruft, der damit vielleicht - schon lange vor den schottischen Clans - die erste Gruppenidentität to go darstellte.

Wenn aber die Energie aus einer sakralen Handlung statt dessen auf dem Schlachtfeld zum Motor des Geschehens wird, so ist damit der „Teamgeist“, das Prinzip des „... wenn zwei oder drei versammelt sind in meinem Namen", das alle Gottheiten hervorgebracht hat, sozusagen entweiht worden. Er ist wieder zum flüchtigen Phänomen regrediert, von der Leine gelassen durch einen mündlichen Appell. Die zu verteidigende Kollektivordnung ist im Krieg bereits etwas Profanes, abgelöst von ihren überirdischen Hütern. Und das auch gerade dann, wenn Götter zur Förderung des Kriegsglücks in die Pflicht genommen werden.

Scheinbar geht es darum, die Ordnung, Inbegriff aller Sicherheit, gegen die Ordnungsfeinde zu verteidigen: eine Gruppe von Solchen, die sich einer andersartigen Ordnung unter-ordnen und damit eine Bedrohung für die unsrige darstellen, von der „wir“ keinen Fußbreit abweichen, nachdem wir unser Blut dafür vergossen haben. So weit, so ehrenhaft. Aber was ist diese Ehrenhaftigkeit? Warum sollte man sein Leben aufs Spiel setzen, um gerade die eine, gewohnte Ordnung zu verteidigen, in die man hineingeboren wurde? Was macht eine Ordnung besser als die andere? Eine fremde Ordnung wird ungeprüft abgelehnt, obwohl augenscheinlich nur das Leben ohne Ordnung für Menschen nicht zu ertragen ist. Zwischen zwei funktionierenden Ordnungen ist der Unterschied, verglichen mit dem Unterschied zwischen Menschen- und Menschenaffendasein, doch verschwindend gering? Und genau da liegt der Haken: Menschen sind sozusagen Kulturpflanzen, die zum Blühen bringen, was man ihnen aufpfropft. Als Kind ist es ihre Aufgabe, sich von der Gruppe in einer Weise erziehen zu lassen, dass sie in eben dieser Gruppe mitspielen können. Sie leben, weil sie zu den Fittesten gehören. Ihr erstes Lächeln, als reizvolle Provokation an das mimetische Vermögen der Artgenossen, ist die Eintrittskarte. Die Welt lächelt zurück. Danach dreht sich der Spieß um und das Kind muss fit werden, in die Welt hineinpassen. Wo aber eine derartige Sozialisierung erst einmal stattgefunden hat, kann es auch eine „Resozialisierung“ geben: man kann, mit Einschränkungen, von neuem lernen, wie man sein muss, wenn man dazugehören will.

Daher die englische Redensart If you can't beat them, join them. Der Slogan des MacDougall Clans, „Sieg oder Tod“, ist demgegenüber gerade dazu da, die join themAlternative von vornherein auszuschließen. Die eigene Gruppe fordert rückhaltlose Hingabe, die mit Mimesis verwandt ist. Diese Hingabe darf in ihrer Reichweite jedoch nur so weit gehen wie die Bannmeile um „uns“. Dieselbe Fähigkeit, den Feinden gegenüber angewendet, würde als Verrat angesehen. Ehrenhaftigkeit ist also nicht viel mehr als ein Mimesis-Tabu: dem Feind 
gegenüber in sich gerade das zu bremsen lernen, was man am besten kann.

Wenn man den Feind attackiert, anstatt mit ihm zu fühlen, scheint Projektion das Gegenteil von Empathie zu sein: die eigene entfesselte Wut spiegelt sich im Gesicht des Gegenübers, so dass beide Seiten eine legitime Veranlassung zum Dreinschlagen haben. Propaganda - das Dazugehören hat mit Zuhören zu tun, so wie Gehorchen mit dem Horchen hat dafür gesorgt, dass das eigene „Monster“ sich an der angeblichen Monstrosität des Gegners orientiert und sich entsprechend in Rage bringt, anstatt die eigene Angst zu spüren und zu wissen: „Die da drüben wollen genauso wenig Schmerzen erleiden wie wir“". Aber wäre eine derartige Einfühlung, das Hineinversetzen in die Verletzbarkeit des anderen, wirklich etwas anderes als Projektion? Ein weiterer Blick zurück auf frühe Rituale hilft dabei, den menschlichen Projektor zu verstehen. Der Teamgeist war über dem Ritual erschienen und das „Team“ hatte daraufhin den „Geist“ zu seinem Gegenüber gemacht, auf den es das eigene Tötenmüssen zu projizieren lernte, an dem es eben deshalb aber auch zugleich sozusagen die eigenen Regungen wiedererkannte. Friedliche Empathie und deren „Feind“, eine Projektion, die Konflikte eskalieren lässt, könnten also auf demselben Mist, dem des Menschenopfers, gewachsen sein.

Der Teamgeist hilft dabei, sich die Ziele der Gruppe anzueignen und als das „einzig Wahre“ anzusehen, seine Kehrseite ist jedoch die Anpassungsfähigkeit an ein nahezu beliebiges Umfeld. Wie erwähnt gibt es gerade aufgrund dieser unerträglichen Leichtigkeit den Hang zu Ehrenhaftigkeit und einem Heldentum, das befiehlt, lieber zu sterben als sich versklaven - oder integrieren - zu lassen. Die Überzeugung, dass es jedem Einzelnen von uns übel ergehen wird, wenn wir die Gruppenidentität, den Teamgeist, preisgeben, ist eine Identifikation mit dem WirGefühl, das Hingabe fordert. Sie kommt einer Identifizierung mit dem Aggressor gleich, denn der gemeinsamen Ordnung hatte man sich einst über mühevolle Jahre hinweg selbst unterordnen müssen. Ein guter Anteil der angeblichen Ordnungsfeindlichkeit der Opponenten ist daher die eigene, die man nicht wahrhaben will und dem Feind in die Schuhe schiebt.

In Slogans wird an das Höhere, die gemeinsamen Ziele, die Nation oder die Herkunft des Teamgeistes appelliert. Ein Beispiel für einen Slogan, der statt dessen mit Einfühlung in den Feind arbeitet, wird in George Orwells Homage to Catalonia erwähnt, worin Orwell eigene Erlebnisse aus dem Spanischen Bürgerkrieg erzählt. Er berichtet, es seien an strategisch günstigen Punkten Männer zum Schreidienst (shouting-duty) postiert worden, die zum Beispiel bedauernswerte Wehrpflichtige, die auf der anderen Seite für die Faschisten kämpfen mussten, mit Slogans wie „Kämpfe nicht gegen deine eigene Klasse!““ zum Desertieren bewegen sollten. 
„The man who did the shouting at the PSUC [Vereinigte Sozialistische Partei Kataloniens, M. M.] post down on our right was an artist at the job. Sometimes, instead of shouting revolutionary slogans he simply told the Fascists how much better we were fed than they were. His account of the Government rations was apt to be a little imaginative. 'Buttered toast!' — you could hear his voice echoing across the lonely valley - 'We're just sitting down to buttered toast over here! Lovely slices of buttered toast!' I do not doubt that, like the rest of us, he had not seen butter for weeks or months past, but in the icy night the news of buttered toast probably set many a Fascist mouth watering. It even made mine water, though I knew he was lying." (Orwell 1989, S. 43 „Homage to Catalonia“)

Streng genommen ist Buttered toast! als Slogan, der wie zufällig die Ohren des hungrigen Gegners erreicht, natürlich gar kein Slogan, denn er richtet sich nicht an die zum Kampf zu motivierenden Freunde. Andererseits hat jeder Schlachtruf diese beiden Seiten, nämlich zum einen die eigenen Truppen zu ermutigen und zugleich den Gegner einzuschüchtern, der den Ruf ja ebenfalls hört, so wie im gälischen Beispiel „Grimmig, wenn gereizt".

Die Behauptung, man verzehre auf der antifaschistischen Seite des Bürgerkrieges gerade Toast mit Butter, vor allem kombiniert mit der Ergänzung „Sogar mir lief das Wasser im Mund zusammen, obwohl ich wusste, dass er log“, schlägt sozusagen eine Brücke zu heutigen Slogans in der Werbung, die ja den potentiellen Konsumenten den Kauf eines Produktes schmackhaft machen sollen, dessen Besitz darum als möglichst erstrebenswert dargestellt wird. Beim Fomulieren und Bebildern versetzt man sich in die Schar der Kaufkräftigen hinein und will sie aus der Reserve locken. Der Slogan verbindet den Krieg mit dem Marketing, indem er den Mehrwert unserer Ordnung beziehungsweise unserer Produkte betont und Loyalität einfordert. Selbst etwas Unsloganhaftes wie Buttered Toast kann bedeuten: „Wir haben uns richtig entschieden, und ihr seid auf der toastlosen Seite“. Die Starbucks Corporation, in der man, Schultz zufolge, ausdrücklich bewusst auf traditionelle Werbung verzichtet und somit nach außen hin keine tatsächlichen Slogans verwendet, kommt den eigenen Mitarbeitern gegenüber nicht ohne einen Schlachtruf aus. Dieser soll sich auf die Gäste nur in sofern auswirken, als das Wir-Gefühl und die damit verbundene, verschwörerische Freundlichkeit zusammen mit dem Kaffee über die Coffee House-Theke gereicht werden soll. Im besten Fall bekommt man dort als Kaffeetrinker die perfekte Mischung aus Zugehörigkeitsgefühl, verbunden mit einem gemeinsamen Stolz auf zum Beispiel das Fair Trade-Siegel und zweitens dem Gefühl, wiedererkannt zu werden und sich Außergewöhnliches wünschen zu dürfen, 
serviert. Milchkaffee im Sessel gibt es ja heute bei jeder zweiten Bäckerei. Nicht so das Starbucks-Erlebnis!

Deshalb: Onward. Vorwärts. Es gibt dafür, um beim Beispiel Starbucks zu bleiben, „5 ways of being“, die Joseph A. Michelli in seinem Buch „The Starbucks Experience“ erklärt. Die vierte von ihnen lautet: „Embrace resistance“. Für Kritik und sogar Widerstand soll man dankbar sein, und lernen, sich darüber $\mathrm{zu}$ freuen. Unter Punkt 4.2 heißt es auch bemerkenswerterweise „Never Try to Beat Them; Always Join Them“, was zum Beispiel bedeutet, dass man den japanischen Markt nicht mit Kaffee beeindrucken konnte, solange man den Menschen dort nicht erklärt hatte, dass es im Bereich Kaffee ähnliche Qualitätsunterschiede gibt wie bei Tee - und für, erstens, Fachwissen und, zweitens, Qualität sei man in Japan weit empfänglicher als für Kaffee.

Die Partner (Starbucks-Sprech für „Mitarbeiter) sollen die 5 ways of being beherzigen, sich aber trotzdem mit ihren besonderen Persönlichkeiten angenommen fühlen und sich mit eigenen Fähigkeiten einsetzen. Es ist bei Schultz die Rede von einer Starbucks-Barista, die eine Datenbank mit den Vornamen aller ihrer Kunden anlegte, bis hin zu einer, die einer Stammkundin eine Niere spendete. Embrace resistance: die Persönlichkeit jedes Einzelnen bietet der Starbucks-Persönlichkeit, der Markenidentität, zunächst Widerstand. Diesem wird genauso begegnet wie dem japanischen Markt voller eingefleischter Teetrinker: mit einer großen, herzlichen Integrationsumarmung, die jeglichen Widerstand erstickt, indem sie ihn gutheißt.

Ein Schelm, wer dabei an die unbarmherzigen Naturgewalten denkt, die menschliche Kollektive ganz zu Anfang aus Verzweiflung „umarmten“, indem sie es ihnen gleich taten und ihrerseits unbarmherzig waren. Das Unternehmen und die Marke scheinen zerbrechlich und schützenswert. Die Marke hat laut Schultz' Buchtitel eine Seele zu verlieren. Darum haben die Mitarbeiter, deren Heil an dem der Marke hängt, allen Grund, ihre Selbstschutzmechanismen auf die Marke zu projizieren, und allen Grund, Opfer zu bringen bis hin zum Spenden einer Niere und den Verzicht auf „Eigengeruch“- bei Starbucks wurden (Schultz 2011, S. 48) "Partner gebeten, kein Parfüm oder Rasierwasser zu tragen, damit das volle Aroma des Kaffees erhalten blieb“. Das große Entgegenkommen, die Anpassungsleistung, scheint laut allgemeiner Darstellung von der Marke zu kommen. Dabei findet die Flucht nach vorn, das hingebungsvolle Sich-eingliedern-Lassen in die Corporation, doch aufseiten der Mitarbeiter statt, während die Marke als eine Art Schutzmacht um jeden Preis unversehrt bleiben und die „Naturgewalt Stock Market" besänftigen muss. 
In der integrierenden Umarmung der Marke wird der Widerspruch aufgelöst. Auch die Kunden sollen davon nicht ausgeschlossen sein. In der Einleitung zu Hermann H. Walas Marketing-Fachbuch (Wala 2011, S. 15) ist zu lesen:

\begin{abstract}
„,Die Marke ist der Geist der Gemeinsamkeit von Unternehmen und Kunden, ein Heimatrevier, das Sinn stiftet', schreibt Publizist Wolf Lotter hellsichtig im Magazin Brand eins. Ausnahmemarken gelingt es, ein Gefühl der Verbundenheit $\mathrm{zu}$ ihren Kunden herzustellen, das über ein bloßes Nutzenversprechen hinausgeht und ihnen langfristige Loyalität sichert. Im Idealfall haben diese Marken Anhänger oder Fans und eben nicht nur ,Käufer‘. Um dieses Wir-Gefühl von Kunden und Marken zu umschreiben, spreche ich von WIR-MARKEN. Wenn es einem Unternehmen gelingt, ein solches Band zu seinen Kunden zu knüpfen, kann ihm der Wettbewerb wenig anhaben. Im Gegenteil: Wenn ,die anderen" wahrgenommen werden, dann um sich davon abzugrenzen und sich der eigenen Identität zu versichern.“
\end{abstract}

Hier taucht also der "Geist der Gemeinsamkeit", schon bekannt unter dem Namen Teamgeist, wieder auf. Das Wahrnehmen der anderen zur bloßen Abgrenzung scheint eine Art Marken-Nationalismus zu sein. Michelli zitiert George Bernard Shaw in „The Starbucks Experience“, im Zusammenhang mit dem ersten Prinzip Make it your own: ,This is the true joy of life, the being used up for a purpose recognized by yourself as a mighty one; being a force of nature instead of a feverish, selfish little clot of ailments and grievances...". Naturgewalt zu sein heißt im Zusammenhang mit Always join them (in den George Bernard Shaw sehr wahrscheinlich nicht hineingehört), alle Entwicklung hin zu einem Subjekt des eigenen Lebens, das mit „Leiden und Beschwerden“ seiner eigenen Natur zu kämpfen hätte, sich dadurch vielleicht ein Ehrgefühl jenseits von Gruppenidentitäten hätte aufbauen können und das sich bereits von der Natur etwas emanzipiert hatte, wieder über Bord zu werfen? Naturgewalt zu sein heißt, sich dem Schrecklichen, Übermächtigen recognized by yourself as a mighty one reflexionslos hinzugeben, wieder ganz wie die ersten Menschen, die darum untereinander zu allem fähig waren und es jederzeit wieder sein werden? Und Naturgewalt zu sein heißt im Fall von Starbucks, dass man Mitarbeiteraktien bekommt, womit man Anteil am Gedeih und Verderb hat, der von den Aktionären ausgeht, ganz wie einst von den Unwettern und wilden Tieren, denen man sich zur Stressbewältigung in Ritualen gleichmachen wollte.

Die Slogans, die der Huldigung einer Marke dienen, sind Schlachtrufe - wie kann es sein, dass allen Akteuren, Kunden genau wie Mitarbeitern und Werbern, vollkommen klar ist, dass sie sich in einer lebenslangen Schlacht befinden, und dass sie sich dennoch die damit verbundenen Gefahren schönreden können? 
Darüber hinaus sind Slogans Volk-Rufe, also sammelnde Rufe. Die erste mögliche Identität in der Menschheitsgeschichte war die, die man durch ein Sich-Identifizieren, das Verschmelzen mit dem Teamgeist, erlangte. Über das Gesammeltwerden lernte man dann, selbst zum Subjekt des Sammelns zu werden, das sich die Erscheinungen der Welt in unterschiedlichste Kategorien, die Tage in Monate und Jahre, das Vieh in Herden einteilte. Bloßes Sammeln macht allerdings genauso „kollektiv einsam“ wie bloßes Gesammeltwerden.

\begin{abstract}
„Nur in der Vermittlung, in der das nichtige Sinnesdatum den Gedanken zur ganzen Produktivität bringt, deren er fähig ist, und andererseits der Gedanke vorbehaltlos dem übermächtigen Eindruck sich hingibt, wird die kranke Einsamkeit überwunden, in der die ganze Natur befangen ist. Nicht in der vom Gedanken unangekränkelten Gewißheit, nicht in der vorbegrifflichen Einheit von Wahrnehmung und Gegenstand, sondern in ihrem reflektierten Gegensatz zeigt die Möglichkeit von Versöhnung sich an. Die Unterscheidung geschieht im Subjekt, das die Außenwelt im eigenen Bewußtsein hat und doch als anderes erkennt. Daher vollzieht sich jenes Reflektieren, das Leben der Vernunft, als bewußte Projektion“ (Horkheimer u. Adorno 2001, S. 198)
\end{abstract}

Auch wenn das Ziel sein sollte, sich nicht in eine Integrationsumarmung, eine zum Teil gemütliche Unmündigkeit zu begeben, wird man also ohne (bewusste) Projektion und vorbehaltlose Hingabe (vonseiten des Gedankens) nicht auskommen, mit der die Menschwerdung einmal angefangen hatte. Jedenfalls liegen die Versöhnung und die Überwindung von Einsamkeit nicht auf dem Grund einer noch so sorgfältig kredenzten Tasse Kaffee, ebenso wenig wie in der Hingabe an eine Geschäftsidee.

Marketingstrategen benutzen darum gerne aus dem militärischen Bereich entlehnte Wörter und Redewendungen, weil sowohl der Krieg als auch das Marketing von der Regression zurück zu einer Gruppenidentität leben. Der Traum von der Nation war ursprünglich der von Bürgerrechten für jeden Einzelnen (wenn auch noch nicht für jede Einzelne) gewesen. Alle emanzipatorischen Bestrebungen haben sich aber bisher als allzu wacklige Übergangsstationen herausgestellt, die den Rückfall in älteste Gewohnheiten nicht verhindern konnten. Und so ist unweigerlich auch das vorgeblich freie Wirkenlassen von Angebot und Nachfrage im Grunde eine Fortsetzung des Krieges mit anderen Mitteln.

\title{
Literatur
}

- Adam, F. (1970). The Clans, Septs \& Regiments Of The Scottish Highlands. London: Johnston and Bacon.

- Horkheimer, M.; Adorno Th. W. (2001). Dialektik der Aufklärung. Philosophische Fragmente. 
Frankfurt/M.: S. Fischer Verlag.

- Orwell, G. (1986). Homage to Catalonia. London: Penguin Books.

- Schultz, H. (2011). Onward. Wie Starbucks erfolgreich ums Überleben kämpfte, ohne seine Seele zu verlieren. Weinheim: Wiley-VCH.

- Türcke, C. (2009). Philosophie des Traums. München: C. H. Beck.

- Wala, H. H. (2012). Meine Marke. Was Unternehmen authentisch, unverwechselbar und langfristig erfolgreich macht. München: Redline Verlag. 